VOL. 47 (1993) [415-426]

\title{
TWIST ORBITS FOR NON CONTINUOUS MAPS OF DEGREE ONE
}

\author{
Francisco Esquembre
}

\begin{abstract}
The existence of twist orbits and twist cycles with a given rotation number is considered for discrete dynamical systems generated by iteration of liftings of maps of the circle into itself. The class of maps for which such orbits exist for every number in the interior of the rotation set is extended to contain an important subclass of non-continuous maps.
\end{abstract}

\section{INTRODUCTION AND STATEMENT OF RESULTS}

Discrete dynamical systems generated by iteration of maps of the circle into itself can be studied using appropriate maps of the real line. If we denote by $p: \mathbb{R} \rightarrow S^{1}$ the projection of the real line onto the circle given by $p(x)=\exp (2 \pi i x)$ (where $i$ denotes here the imaginary unity), we say that a map $F: \mathbb{R} \rightarrow \mathbb{R}$ is a lifting of a map $f: S^{1} \rightarrow S^{1}$, if $p \circ F=f \circ p$ and there is a $k \in \mathbb{Z}$ such that $F(x+1)=F(x)+k$, for every $x \in \mathbb{R}$. This $k$ is called the degree of the lifting $F$.

We shall consider in this paper maps with liftings of degree one, since the corresponding systems have been found to exhibit the most interesting dynamic properties (see [1]). Hence, a map $F: \mathbb{R} \rightarrow \mathbb{R}$ is said to be of degree one if $F(x+1)=F(x)+1$, for every $x \in \mathbb{R}$.

The notions of orbit and cycle for maps of the circle extend in a natural way as follows. We call $\bigcup_{n \geqslant 0}\left(F^{n}(x)+\mathbb{Z}\right)$ the orbit $\bmod 1$ of $x$ under $F$ the set. We say that $x$ is a periodic mod 1 point or, equivalently, the orbit of $x$ is a cycle mod 1 , of period $q \in \mathbb{N}$ and rotation number $p / q$, if $F^{q}(x)-x=p \in \mathbb{Z}$ and $F^{1}(x)-x \notin \mathbb{Z}$ for $i=1,2, \ldots, q-1$.

For any orbit mod 1 , the rotation number can be defined, whenever the sequence $\left(\left(F^{n}(x)-x\right) / n\right)_{n \in N}$ converges, as the number $\rho_{F}(x)=\lim \left(F^{n}(x)-x\right) / n$. It is easy to see (see Lemma 2) that for a cycle mod 1 of period $q$ and rotation number $p / q$, this limit exists and equals precisely $p / q$, hence both definitions coincide.

The set containing all rotation numbers is denoted by $\operatorname{Rot}(F)$ and is called the rotation set of $F$. It contains information about the long term behaviour of all the orbits mod 1 which have rotation number. Whenever $\operatorname{Rot}(F)$ reduces to one point, we

Received 12 May 1992

Copyright Clearance Centre, Inc. Serial-fee code: 0004-9729/93 \$A2.00+0.00. 
denote it by $\rho(F)$ and call it the rotation number of $F$. This is precisely the case for every non-decreasing map of degree one (see [10]).

For continuous maps of degree one the rotation set is a closed interval (see [7]), and provides important information about the existence and behaviour of orbits mod 1 (see [9] and [2]). Easier proofs of some of these facts were given in [3], using the notion of twist orbit. An orbit mod 1 is said to be twist if the map restricted to this orbit is non-decreasing. Twist orbits turn out to be of great value in studying continuous systems, since they can be effectively used to compute lower bounds of the topological entropy (see for instance [1]). This is based on the fact that, for continuous maps of degree one, twist orbits exist for every number in the rotation set and, if the number is rational, then twist cycles also exist (see [3]).

There are some problems, however, for which non-continuous liftings of degree one are of interest, for instance, when taking liftings of some monotone mod 1 maps (see [6]) or when studying Newton's method of finding zeros of certain functions (see [8]). In a previous work (see [5]), it was shown that for an important class of non-necessarily continuous maps the rotation set is still a closed interval, and a powerful tool for the study the of the existence and behaviour of orbits mod 1 .

The goal of this paper is to show that for a class of maps of degree one containing, but not restricted to, continuous maps, orbits (respectively cycles) mod 1 also exist for every number (respectively rational number) in the interior of the rotation set.

Let us now introduce some notation. We shall denote by $\mathcal{B}_{1}$ the class of all the maps $F: \mathbb{R} \rightarrow \mathbb{R}$ of degree one that are bounded on $[0,1]$. Note that this includes non-decreasing maps of degree one. In this class, we consider the topology induced by the distance $d(F, G)=\sup \{|F(x)-G(x)|: x \in \mathbb{R}\}$. For any map $F$ of $\mathcal{B}_{1}$, we construct the new maps $F_{\ell}(x)=\inf \{F(y): y \geqslant x\}$ and $F_{u}(x)=\sup \{F(y): y \leqslant x\}$. These are two non-decreasing maps of $\mathcal{B}_{1}$ (see Lemma 4) lying immediately below and sitting immediately above of the original map, respectively.

Given a map $F$ of degree one and $x \in \mathbb{R}$, we denote by $F(x+)$ the limit $\lim _{y \rightarrow x^{+}} F(y)$ and by $F\left(x^{-}\right)$the limit $\lim _{y \rightarrow x^{-}} F(y)$, whenever these limits exist. We also introduce the symbols $F(x \circ)=F(x)$ and $F(x$ ?). This last symbol is a wild card character meaning any of the symbols $F(x-), F(x \circ)$ or $F(x+)$. Finally, we denote by Cont $(F)$ the set of all points of continuity of $F$, by $\operatorname{Disc}(F)$ its complement and by Const $(F)$ the set of all points $x$ such that $F$ is constant in $(x-\varepsilon, x+\varepsilon)$ for some $\varepsilon>0$.

Let $F$ be a map of $\mathcal{B}_{1}$ such that $F(x-)$ and $F(x+)$ exist for every $x \in \mathbb{R}$, and such that for every point $x$ of discontinuity of $F$ the following is satisfied:

(I) $F(x-)>F(x+)$,

(II) if $F(x)>F(x-)$ then there exist $c<x$ and ? $\in\{+,-$, o $\}$ such that 


$$
F(c ?) \geqslant F(x) \text { and } F_{\ell}(c ?)=F_{\ell}(x)
$$

(III) if $F(x+)>F(x)$ then there exist $c>x$ and ? $\in\{+,-$, o $\}$ such that $F(x) \geqslant F(c ?)$ and $F_{u}(x)=F_{u}(c ?)$.

Under these conditions, $F_{\ell}$ and $F_{u}$ are continuous at every point of $\mathbb{R}$ (see Lemma 6 ) and Theorem A of [5] yields $\operatorname{Rot}(F)=\left[\rho\left(F_{\ell}\right), \rho\left(F_{u}\right)\right]$.

Given any $x \in \mathbb{R}$, we define the functions $\ell(x)=\inf \left\{y \leqslant x:(y, x) \subset\right.$ Const $\left.\left(F_{u}\right)\right\}$ and $r(x)=\sup \left\{y \geqslant x:(x, y) \subset\right.$ Const $\left.\left(F_{\ell}\right)\right\}$. Clearly, $\ell$ and $r$ are non-decreasing real functions, $\ell(x) \leqslant x \leqslant r(x)$ for every $x \in \mathbb{R}$ and, under our hypotheses, $F_{u}(\ell(x))=$ $F(\ell(x)-)$ and $F_{\ell}(r(x))=F(r(x)+)$. Let $D$ denote the set $\{d \in \operatorname{Disc}(F): \ell(d) \notin$ Cont $(F)$ or $\left.F_{\ell}(\ell(d)) \neq F_{1}(d)\right\}$.

The main result of this paper is the following theorem.

Theorem 1. Under these hypotheses, if $D \cap[0,1]$ is a finite set then, for any $r \in\left(\rho\left(F_{1}\right), \rho\left(F_{u}\right)\right), F$ has a twist orbit with rotation number $r$. If $r$ is a rational number, then $F$ has a twist cycle mod 1 with rotation number $r$.

To our knowledge this result establishes the largest known class for which this property holds. Note also that, in general, this result cannot be improved, in the sense that the property might not hold for the ends of the rotation interval, as the map in the following picture shows.

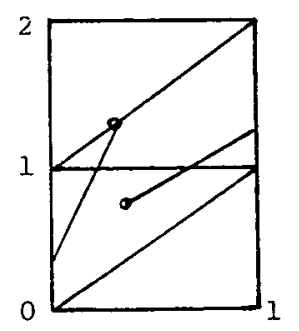

Graph of $F$ on $[0,1]$

$F_{u}$ has a cycle of rotation number 1 (and hence 1 belongs to the rotation set of $F$ ) but $F$ has not. However, with additional conditions, this can be true also for some non-continuous maps of degree one (see [4]).

The rest of this paper is devoted to the proof of Theorem 1.

\section{Preliminary Results}

We first state and prove some auxiliary results. For the sake of completeness we also include the proofs of some well known results.

Lemma 2. Let $F: \mathbb{R} \rightarrow \mathbb{R}$ be a map of degree one, $x \in \mathbb{R}, p \in \mathbb{Z}$ and $q \in \mathbb{N}$.

(2.a) If $F^{q}(x)=x+p$ then $\rho_{F}(x)=p / q$. 
If $F$ is non-decreasing, then the following also holds:

(2.b) $\rho_{F}(x)$ exists, belongs to $\mathbb{R}$ and is independent of $x$.

(2.c) If $F^{q}(x) \geqslant x+p$, then $\rho(F) \geqslant p / q$.

(2.d) If $F^{q}(x) \leqslant x+p$, then $\rho(F) \leqslant p / q$.

(2.e) If $F$ has an orbit contained in Cont $(F)$, then the map $\rho$, defined from the set of all non-decreasing maps of degree one into $\mathbb{R}$, is continuous at $F$.

(2.f) If $F$ is continuous, then $\rho(F) \in \mathbb{Q}$ if and only if $F$ has a cycle mod 1 .

Proof: (2.b) is Theorem 1 of [10] and we omit the proof. If $F^{q}(x)=x+p$, then for any given $n \in \mathbb{N}$, we set $n=k q+i, 0 \leqslant i \leqslant q-1$, and obtain $F^{n}(x)=F^{i}\left(F^{k q}(x)\right)=$ $F^{i}\left(F^{(k-1) q}(x)+p\right)=F^{i}\left(F^{(k-2) q}(x)+2 p\right)=\ldots=F^{i}(x+k p)=F^{i}(x)+k p$. From this $\left(F^{n}(x)-x\right) / n=\left(F^{i}(x)+k p-x\right) /(k q+i)$, where $k$ tends to infinity whenever $n$ tends to infinity and the other quantities remain bounded, therefore $\left(F^{n}(x)-x\right) / n$ converges ot $p / q$. This proves (2.a).

If $F^{q}(x) \geqslant x+p$ then $F^{k q}(x) \geqslant x+k p$, for all $k \in \mathbb{N}$, hence $\left(F^{k q}(x)-x\right) /(k q) \geqslant$ $p / q$ and $\rho_{F}(x)=\rho(F) \geqslant p / q$; this gives (2.c). (2.d) can be proved similarly.

In order to prove (2.e), given any $\varepsilon>0$ we look for $\delta>0$ such that for every non-decreasing map $G$ of $\mathcal{B}_{1}, d(F, G) \leqslant \delta$ implies $|\rho(F)-\rho(G)| \leqslant \varepsilon$. Given $\varepsilon$ take $p \in \mathbb{Z}, q \in \mathbb{N}$ such that $\rho(F)-\varepsilon \leqslant p / q<\rho(F)$. This gives $F^{q}(x)>x+p$, for every $x \in \mathbb{R}$. Since $F$ has a point $x_{0}$ whose orbit is contained in $\operatorname{Cont}(F)$, then $F$ is left continuous at $F^{i}\left(x_{0}\right), 0 \leqslant i \leqslant q$, that is for every $\varepsilon_{i}>0$ there exists $\delta_{i}>0$ such that $F^{i}\left(x_{0}\right)-\delta_{i} \leqslant z$ implies $F^{i+1}\left(x_{0}\right)-\varepsilon_{i} \leqslant F(z)$. In particular $F^{i+1}\left(x_{0}\right)-\varepsilon_{i} \leqslant$ $F\left(F^{i}\left(x_{0}\right)-\delta_{i}\right)$. Consider $\varepsilon_{q-1}=\left(F^{q}\left(x_{0}\right)-x_{0}-p\right) / 2>0, \varepsilon_{i-1}=\delta_{i} / 2,2 \leqslant i \leqslant q-1$, and take $\delta=\min \left(\delta_{1}, \delta_{2} / 2, \ldots, \delta_{q-1} / 2, \varepsilon_{q-1}\right)>0$. Now, if $G$ verifies $d(F, G) \leqslant \delta$, then $F(x)-\delta \leqslant G(x)$, for every $x \in \mathbb{R}$, hence $F\left(x_{0}\right)-\delta_{1} \leqslant G\left(x_{0}\right)$ and $F\left(F\left(x_{0}\right)-\delta_{1}\right)-$ $\delta \leqslant G^{2}\left(x_{0}\right)$. This gives $F\left(F\left(x_{0}\right)-\delta_{1}\right)-\delta_{2} / 2 \leqslant G^{2}\left(x_{0}\right)$ and, from here, $F^{2}\left(x_{0}\right)-\delta_{2} \leqslant$ $G^{2}\left(x_{0}\right)$. Repeating this reasoning we obtain that $F^{q-1}\left(x_{0}\right)-\delta_{q-1} \leqslant G^{q-1} \leqslant G^{q-1}\left(x_{0}\right)$ implies $F\left(F^{q-1}\left(x_{0}\right)-\delta_{q-1}\right)-\varepsilon_{q-1} \leqslant G^{q}\left(x_{0}\right)$, which gives $F^{q}\left(x_{0}\right)-2 \varepsilon_{q-1} \leqslant G^{q}\left(x_{0}\right)$ and therefore, $x_{0}+p \leqslant G^{q}\left(x_{0}\right)$ and $\rho(G) \geqslant p / q \geqslant \rho(F)-\varepsilon$. Similarly one can find $\delta^{\prime}>0$ such that $d(F, G) \leqslant \delta^{\prime}$ implies $\rho(G) \leqslant \rho(F)+\varepsilon$.

The condition in (2.f) is sufficient because of (2.a), we now show that it is also necessary. Assume that the equation $F^{q}(x)=x+p$ has no solution for any $p \in \mathbb{Z}$ and $q \in \mathbb{N}$. Since $F$ is continuous, so is $F^{q}-\mathrm{Id}-p$, and we can divide $\mathbb{Q}$ into two classes,

$$
\begin{aligned}
& A_{1}=\left\{p / q:(p, q)=1, F^{q}(x)>x+p, \text { for all } x \in \mathbb{R}\right\} \\
& A_{2}=\left\{p / q:(p, q)=1, F^{q}(x)<x+p, \text { for all } x \in \mathbb{R}\right\}
\end{aligned}
$$

(here $(p, q)$ denotes the greatest common divisor of $p$ and $q$ ). If $(p, q)=(r, s)=1$, $p / q \in A_{1}$ and $r / s \in A_{2}$, we show that for any $x \in \mathbb{R}, p / q<\rho_{F}(x)<r / s$. Since 
$F$ is continuous there exists $\delta>0$ such that $F^{q}(x)-x \geqslant p+\delta$, for all $x \in \mathbb{R}$. Let $m=\inf \left\{F^{i}(x)-x: x \in \mathbb{R}, 0 \leqslant i \leqslant q-1\right\}$. Since $n \geqslant 0$ then $n=k q+i, 0 \leqslant i \leqslant q-1$ and

$$
\begin{aligned}
F^{n}(x)-x & =\left[F^{i}\left(F^{k q}(x)\right)-F^{k q}(x)\right]+\left[F^{k q}(x)-x\right] \\
& =\left[F^{i}\left(F^{k q}(x)\right)-F^{k q}(x)\right]+\sum_{j=0}^{k-1}\left[F^{q}\left(F^{j q}(x)\right)-F^{j q}(x)\right] \\
& \geqslant m+k(p+\delta)>m+(n / q-1)(p+\delta) .
\end{aligned}
$$

Hence, dividing by $n$ and taking limits, we obtain $\rho_{F}(x) \geqslant(p+\delta) q>p / q$. The other inequality can be proved similarly. Therefore $A_{1}$ is a Dedekind cut (see [11]) and there exists $\rho \in \mathbb{R}$ such that $\rho_{F}(x)=\rho$ and $\rho \notin A_{1} \cup A_{2}=\mathbb{Q}$.

Lemma 3. Let $F: \mathbb{R} \rightarrow \mathbb{R}$ be a continuous map of degree one and $k \in \mathbb{N}$. Then the following hold:

(3.a) $\bigcup_{i=0}^{k-1} F^{-i}(\operatorname{Const}(F)) \subseteq$ Const $\left(F^{k}\right)$.

(3.b) $F$ has an orbit disjoint from Const $(F)$.

(3.c) If $F$ is non-decreasing, then $\rho(F) \in \mathbb{Q}$ if and only if $F$ has a cycle mod 1 disjoint from Const $(F)$.

Proof: If $x_{0} \in F^{-i}$ (Const $\left.(F)\right)$ for some $0 \leqslant i \leqslant k-1$, then $F^{i}\left(x_{0}\right) \in$ Const $(F)$, that is, there exists $\varepsilon>0$ such that $F(y)=F^{i+1}\left(x_{0}\right)$, for all $\left|y-F^{i}\left(x_{0}\right)\right|<\varepsilon$. Since $F^{i}$ is continuous, there is a $\delta>0$ with $\left|F^{i}(x)-F^{i}\left(x_{0}\right)\right|<\varepsilon$, for every $\left|x-x_{0}\right|<\delta$; then $F^{i+1}(x)=F^{i+1}\left(x_{0}\right)$, for every $\left|x-x_{0}\right|<\delta$, hence $F^{k}(x)=F^{k}\left(x_{0}\right)$, for every $\left|x-x_{0}\right|<\delta$ and, from this, $x_{0} \in$ Const $\left(F^{k}\right)$. This proves (3.a).

We now show (3.b). Since Const $(F)$ is an open set, the sequence $A_{n}=$ $\bigcup_{i=0}^{n-1} F^{-i}($ Const $(F))$ is a non-decreasing sequence of open sets. Suppose $[0,1] \subseteq \bigcup_{n=1}^{\infty} A_{n}$, then, since $[0,1]$ is a compace set, there would exist $k \in \mathbb{N}$ with $[0,1] \subseteq \bigcup_{n=1}^{k} A_{n}$ and (since $\left.A_{n}+\mathbb{Z}=A_{n}\right) \mathbb{R}=\bigcup_{n=1}^{k} A_{n}=A_{k}$. Hence $\mathbb{R}=\bigcup_{i=0}^{k-1} F^{-i}($ Const $(F)) \subseteq$ Const $\left(F^{k}\right)$ would imply $F^{k}$ is constant on $\mathbb{R}$ and $F^{k}(x+1)=F^{k}(x)$, which contradicts the fact that $F$ is of degree one. Therefore there exists $x \in[0, q]-\bigcup_{n=1}^{\infty} A_{n}$, and $x \notin \bigcup_{i=0}^{\infty} F^{-i}$ (Const $\left.(F)\right)$ has the desired orbit.

The condition in (3.c) is sufficient due to (2.f). If $\rho(F)=p / q \in \mathbb{Q}$, then the proof of (2.f) gives the existence of $z \in \mathbb{R}$, a fixed point of $G=F^{q}-p$. Since $F$ is of degree one, $z+1$ is also a fixed point for $G$ and, since $F$ is non-decreasing, so is $G$. Now (3.b) proves $F$ has an orbit, starting in a point $x$, disjoint from Const $(F)$, and we 
can assume without loss of generality that $x \in[z, z+1]$. The sequence $\left(G^{n}(x)\right)_{n=0}^{\infty}$ is non-decreasing and bounded with limit $y \in \mathbb{R}$ satisfying $G(y)=y$. Since Const $(F)$ is an open set, $\bigcup_{i=0}^{\infty} F^{-i}($ Const $(F))$ is also open and the points $G^{n}(x)$ have orbits under $F$ disjoint from Const $(F)$, which implies $y$ has its orbit disjoint from Const $(F)$ and is therefore the desired cycle mod 1.

Lemma 4. Let $F \in \mathcal{B}_{1}$ and let $F_{\ell}, F_{u}$ be the maps defined in Section 1.

(4.a) $F_{\ell}$ and $F_{u}$ are non-decreasing maps of $\mathcal{B}_{1}$.

(4.b) $\quad F_{\ell}(x) \leqslant F(x) \leqslant F_{u}(x)$, for every $x \in \mathbb{R}$.

(4.c) The maps defined by $F \mapsto F_{\ell}, F \mapsto F_{u}$ are non-decreasing and Lipschitz continuous.

(4.d) If $F$ is non-decreasing then $F=F_{\ell}=F_{u}$.

(4.e) Const $(F) \subseteq$ Const $\left(F_{\ell}\right) \cap \operatorname{Const}\left(F_{u}\right)$.

(4.f) $\operatorname{Cont}(F) \subseteq \operatorname{Cont}\left(F_{\ell}\right) \cap \operatorname{Cont}\left(F_{u}\right)$.

(4.g) Let $x_{0} \in$ Cont $(F)$, then

(4.g.a) $F_{l}\left(x_{0}\right) \neq F\left(x_{0}\right) \Rightarrow x_{0} \in \operatorname{Const}\left(F_{l}\right)$

(4.g.b) $F_{u}\left(x_{0}\right) \neq F\left(x_{0}\right) \Rightarrow x_{0} \in$ Const $\left(F_{u}\right)$.

ProOF: The proofs of (4.b), (4.d) and (4.e) are straightforward. Now, note that if $x \leqslant x^{\prime}$ then $\{F(y): y \leqslant x\} \subseteq\left\{F(y): y \leqslant x^{\prime}\right\}$, hence $F_{u}(x) \leqslant F_{u}\left(x^{\prime}\right)$ and similarly for $F_{\ell}$. Also, $F_{u}(x+1)=\sup \{F(y): y \leqslant x+1\}=\sup \{F(z+1): z \leqslant x\}=\sup \{F(z)+$ $1: z \leqslant x\}=F_{u}(x)+1$. Let $m, M \in \mathbb{R}$ be such that $m \leqslant F(x) \leqslant M$, for all $x \in[0,1]$. For any $x \in[0,1]$ and given any $y \leqslant x$, there exist $y^{\prime} \in[0,1], k \in \mathbb{N}$ such that $y=y^{\prime}-k ;$ this leads to $F(y)=F\left(y^{\prime}-k\right)=F\left(y^{\prime}\right)-k \leqslant M-k \leqslant M$. This and $F(x) \leqslant F_{u}(x)$ yield $m \leqslant F_{u}(x) \leqslant M$ for all $x \in[0,1]$, hence $F_{u}$ is a non-decreasing map of $\mathcal{B}_{1}$. Similarly for $F_{\ell}$.

If $F(x) \leqslant G(x)$ for all $x \in \mathbb{R}$, then for every $x \in \mathbb{R}$ and $y \leqslant x, F(y) \leqslant G(y)$; hence $F_{u}(x) \leqslant G_{u}(x)$ for all $x \in \mathbb{R}$. Let $F, G$ be two maps of $\mathcal{B}_{1}$ and set $d=\|F-G\|_{\infty}$; we show that for every $x \in \mathbb{R},\left|F_{u}(x)-G_{u}(x)\right| \leqslant d$. For this, suppose there exist $x \in \mathbb{R}$ and $\varepsilon>0$ such that $F_{u}(x)-G_{u}(x)>d+\varepsilon$. Then there is a $y \leqslant x$ with $F(y)>F_{u}(x)-\varepsilon$, hence $F(y)-G(y)>F_{u}(x)-\varepsilon-G_{u}(x)>d$, which is not possible. We proceed similarly for $F_{\ell}$. This proves (4.c).

Let $x_{0} \in \operatorname{Cont}(F)$; we prove left continuity of $F_{u}$ and $F_{\ell}$ at $x_{0}$. The right continuity can be proved similarly, and (4.f) follows from both. Given any $\varepsilon>0$, there exist $\delta>0$ such that $x_{0}-\delta \leqslant z \leqslant x_{0}$ implies $F\left(x_{0}\right)-\varepsilon \leqslant F(z) \leqslant F\left(x_{0}\right)+\varepsilon$. Let $x_{0}-\delta<x<x_{0}$, then $\left|F_{u}\left(x_{0}\right)-F_{u}(x)\right|=F_{u}\left(x_{0}\right)-F_{u}(x)=\sup \left\{F(y): y \leqslant x_{0}\right\}-$ $\sup \{F(y): y \leqslant x\}$. Now, $\sup \left\{F(y): y \leqslant x_{0}\right\} \leqslant \max \left(\sup \{F(y): y \leqslant x\}, F\left(x_{0}\right)+\varepsilon\right)$ and $\sup \{F(y): y \leqslant x\} \geqslant F(x) \geqslant F\left(x_{0}\right)-\varepsilon$. From both, $\left|F_{u}\left(x_{0}\right)-F_{u}(x)\right| \leqslant$ $\max \left(0, F\left(x_{0}\right)+\varepsilon-\sup \{F(y): y \leqslant x\}\right) \leqslant 2 \varepsilon$. Similarly, $\left|F_{\ell}\left(x_{0}\right)-F_{\ell}(x)\right|=$ 
$\inf \left\{F(y): y \geqslant x_{0}\right\}-\inf \{F(y): y \geqslant x\} ; \operatorname{since} \inf \{F(y): y \geqslant x\} \geqslant \min \left(\inf \left\{F(y): y \geqslant x_{0}\right\}\right.$, $\left.F\left(x_{0}\right)-\varepsilon\right)$, we obtain $\left|F_{\ell}\left(x_{0}\right)-F_{\ell}(x)\right| \leqslant \max \left(0, \inf \left\{F(y): y \geqslant x_{0}\right\}-F\left(x_{0}\right)+\varepsilon\right) \leqslant \varepsilon$.

In order to prove (4.g), suppose $F_{u}\left(x_{0}\right) \neq F\left(x_{0}\right)$. Then $F_{u}\left(x_{0}\right)>F\left(x_{0}\right)$ and there exists $y<x_{0}$ with $F(y)>F\left(x_{0}\right)$. Since $F$ is continuous at $x_{0}$ there exists $\varepsilon>0$ such that $F(x)<F(y)$, for every $\left|x-x_{0}\right|<\varepsilon$. Hence $F_{u}(x)=\sup \left\{F(z): z \leqslant x_{0}-\varepsilon\right\}$, for every $\left|x-x_{0}\right|<\varepsilon$ and $x_{0} \varepsilon$ Const $\left(F_{u}\right)$. Similarly for $F_{\ell}$.

Lemma 5. Let $F \in \mathcal{B}_{1}, x_{0} \in \mathbb{R}$ and $? \in\{0,+,-\}$.

(5.a) If there exist $c<x_{0}, \varepsilon>0$ such that $F(c$ ? $) \geqslant F(x)$ for every $\left|x-x_{0}\right|<$ $\varepsilon$, then $x_{0} \in$ Const $\left(F_{u}\right)$.

(5.b) If there exist $c>x_{0}, \varepsilon>0$ such that $F(x) \geqslant F\left(c\right.$ ?) for every $\left|x-x_{0}\right|<$ $\varepsilon$, then $x_{0} \in$ Const $\left(F_{l}\right)$.

(5.c) $x_{0} \in \operatorname{Cont}\left(F_{u}\right)$ if any of the following conditions is satisfied:

(5.c.1) $x_{0} \in \operatorname{Cont}(F)$

(5.c.2) $x_{0} \in$ Const $\left(F_{u}\right)$

(5.c.3) $F\left(x_{0}-\right)=F\left(x_{0}+\right)>F\left(x_{0}\right)$

(5.c.4) $F\left(x_{0}-\right)>F\left(x_{0}+\right), F\left(x_{0}-\right) \geqslant F\left(x_{0}\right)$.

(5.d) $x_{0} \in \operatorname{Cont}\left(F_{\ell}\right)$ if any of the following conditions is satisfied:

(5.d.1) $x_{0} \in \operatorname{Cont}(F)$

(5.d.2) $x_{0} \in$ Const $\left(F_{\ell}\right)$

(5.d.3) $F\left(x_{0}\right)=F\left(x_{0}+\right)<F\left(x_{0}\right)$

(5.d.4) $F\left(x_{0}-\right)>F\left(x_{0}+\right), F\left(x_{0}\right) \geqslant F\left(x_{0}+\right)$.

Proof: We first prove (5.a), (5.b) follows in a similar way. We can take $c<x_{0}-\varepsilon$. If $?=0$ or $?=-$ then clearly, $F_{u}(c) \geqslant F\left(c\right.$ ?). If $?=+$, take $c<y<x_{0}-\varepsilon$ and we get $F_{u}(y) \geqslant F(c+) \geqslant F\left(c\right.$ ?). So, in every case, there exists $y<x_{0}-\varepsilon$ such that $F_{u}(y) \geqslant$ $F(x)$, for every $\left|x-x_{0}\right|<\varepsilon$. Now, $\left|x-x_{0}\right|<\varepsilon$ implies $F_{u}(x)=\sup \{F(z): z \leqslant x\}=$ $\max \left(\sup \left\{F(z): x_{0}-\varepsilon<z \leqslant x\right\}, \sup \left\{F(z): z \leqslant x_{0}-\varepsilon\right\}\right)=\sup \left\{F(z): z \leqslant x_{0}-\varepsilon\right\}=$ $F_{u}\left(x_{0}-\varepsilon\right)$; hence $x_{0} \in$ Const $\left(F_{u}\right)$.

If (5.c.1) or (5.c.2) is satisfied then (5.c) follows trivially. Note that $F\left(x_{0}-\right) \geqslant$ $F\left(x_{0}\right)$ implies that $\sup \left\{F(y): y \leqslant x_{0}\right\}=\sup \left\{F(y): y<x_{0}\right\}=\sup \{\sup \{F(y): y \leqslant$ $\left.x\}: x<x_{0}\right\}=\sup \left\{F_{u}(x): x<x_{0}\right\}$. If (5.c.3) is satisfied then $F\left(x_{0}-\right)>F\left(x_{0}\right)$ gives $\sup \left\{F_{u}(x): x<x_{0}\right\}=F_{u}\left(x_{0}\right)$ and $F_{u}\left(x_{0}\right)=\sup \left\{F(y): y<x_{0}\right\}=\inf \{\sup \{F(y): y \leqslant$ $\left.x\}: x>x_{0}\right\}=\inf \left\{F_{u}(x): x>x_{0}\right\}$, since $F\left(x_{0}-\right)=F\left(x_{0}+\right)$; from both identities we obtain that $F_{u}$ is continuous at $x_{0}$. Finally (5.c.4) implies $F\left(x_{0}-\right) \geqslant F\left(x_{0}\right)$ which again gives that $F_{u}$ is left continuous at $x_{0}$. Also, $F\left(x_{0}-\right)>F\left(x_{0}+\right)$ gives the existence of $\varepsilon>0$ such that $F\left(x_{0}-\right)>F(x)$, for every $x_{0}<x<x_{0}+\varepsilon$. Hence there is a $y<x_{0}$ such that $F(y)>F(x)$, for every $x_{0}<x<x_{0}+\varepsilon$ and from here $F_{u}(x)=F_{u}\left(x_{0}\right)$, for every $x_{0}<x<x_{0}+\varepsilon$ and $\inf \left\{F_{u}(x): x<x_{0}\right\}=F_{u}\left(x_{0}\right)$. (5.d) can be proved 
similarly.

LEMMA 6. Let $F \in \mathcal{B}_{1}$ such that $F(x-)$ and $F(x+)$ exist for every $x \in \mathbb{R}$, and let $x_{0} \in \mathbb{R}$. If there exists $? \in\{0,+,-\}$ such that any of the following conditions hold:

(6.1) there exists $c<x_{0}$ such that $F(c$ ? $) \geqslant F\left(x_{0}-\right) \geqslant R\left(x_{0}+\right)$

(6.2) $F\left(x_{0}-\right) \geqslant F\left(x_{0}\right) \geqslant F\left(x_{0}+\right)$

(6.3) there exists $C>x_{0}$ such that $F\left(x_{0}-\right) \geqslant F\left(x_{0}+\right)>F\left(x_{0}\right) \geqslant R(c$ ?)

then $F_{\ell}$ and $F_{u}$ are continuous at $x_{0}$ and satisfy:

(6.a) if $x_{0} \in \mathbb{R}-$ Const $\left(F_{\ell}\right)$ then $F_{\ell}\left(x_{0}\right)=F\left(x_{0}+\right)$

(6.b) if $x_{0} \in \mathbb{R}-$ Const $\left(F_{u}\right)$ then $F_{u}\left(x_{0}\right)=F\left(x_{0}-\right)$.

Proof: The continuity of $f_{\ell}$ and $F_{u}$ at $x_{0}$ follows from Lemma 5. We prove (6.1). (6.b) can be proved analogously. If (6.3) is satisfied then the case is trivial because it implies, by (5.b), that $x_{0} \in$ Const $\left(f_{\ell}\right)$. If (6.1) or (6.2) hold then $F_{\ell}\left(x_{0}\right) \neq F\left(x_{0}+\right)$ gives $F_{\ell}\left(x_{0}\right)<F\left(x_{0}+\right) \leqslant \min \left(f\left(x_{0}-\right), F\left(x_{0}\right)\right)$. Hence there exists $\varepsilon>0, y>x_{0}$ such that $F(x)>F(y)$, for every $\left|x-x_{0}\right|<\varepsilon$; therefore $x_{0} \in \operatorname{Const}\left(F_{\ell}\right)$.

\section{Proof of Theorem 1}

We give the proof as the consequence of a series of results. Set $m=\min \{F(d-)-$ $F(d+): d \in D\}$ and $T=\sup \left\{F_{u}(x)-F_{\ell}(x): x \in \mathbb{R}\right\}$. Clearly $T \geqslant m \geqslant 0$, with $m>0$ if $D \neq 0$. Consider the map $f$ defined from $[0, T]$ into $\mathbb{R}$ by

$$
f(t)= \begin{cases}0 & \text { if } t<T-m / 2 \\ t-T+m / 2 & \text { if } t \geqslant T-m / 2\end{cases}
$$

$f$ is a non-decreasing continuous map satisfying $f(t) \geqslant 0$ for all $t, f(0)=0$ and $f(T)=m / 2$.

We define the families of maps $\left\{L_{t}\right\}_{t \in[0, T]},\left\{M_{t}\right\}_{t \in[0, T]}$ and $\left\{N_{t}\right\}_{t \in[0, T]}$ as follows,

$$
\begin{aligned}
L_{t}(x) & = \begin{cases}F_{\ell}(x)+t+f(t) & \text { if }[x, r(x)] \cap D=\emptyset \\
\min \left(F_{\ell}(x)+t, F(d-)-m / 2\right)+f(t) & \text { if } d=\min ([x, r(x)] \cap D)\end{cases} \\
M_{t}(x) & =\min \left(F(x+), L_{t}(x)\right) \\
N_{t}(x) & =\left(M_{t}\right)_{u}
\end{aligned}
$$

for every $t \in[0, T]$. The next results show important properties of the family $\left\{N_{t}\right\}$.

LEMma 7. $\left\{N_{t}\right\}_{t \in[0, T]}$ is a family of non-decreasing maps of degree one of $\mathbb{R}$ into itself. The map $t \mapsto N_{t}$ is non-decreasing and Lipschitz continuous. Also, $N_{0}=F_{\ell}$ and $N_{T}=F_{u}$.

Proof: Since the sets $D$ and Const $\left(F_{\ell}\right)$ are invariant modulo 1 and $F \in \mathcal{B}_{1}$, we have that $L_{t}$ and $M_{t}$ belong to $\mathcal{B}_{1}$ for every $t \in[0, T]$. Using Lemma 4 we 
obtain that $N_{t} \in \mathcal{B}_{1}$ and that $N_{t}$ is non-decreasing for every $t \in[0, T]$. Given any $t_{1}$ and $t_{2} \in \mathbb{R}$, if $t_{1} \leqslant t_{2}$ then trivially $M_{t_{1}} \leqslant M_{t_{2}}$ and, by (4.c), $N_{t_{1}} \leqslant N_{t_{2}}$. Also, $\left\|N_{t_{1}}-N_{t_{2}}\right\| \leqslant\left\|M_{t_{1}}-M_{t_{2}}\right\| \leqslant\left\|L_{t_{1}}-L_{t_{2}}\right\| \leqslant 2\left|t_{1}-t_{2}\right|$.

Take $t=0$ and $x \in \mathbb{R}$. If $[x, r(x)] \cap D=\emptyset$ then $L_{0}(x)=F_{\ell}(x)$. Since $F(d-)-m / 2 \geqslant F(d+)$ for all $d \in D$, if $[x, r(x)] \cap \neq \emptyset$ and $d=\min ([x, r(x)] \cap D)$ then $L_{0}(x)=\min \left(F_{\ell}(x), F(d-)-m / 2\right)=F_{\ell}(x)$. Thus, in both cases $M_{0}(x)=$ $\min \left(F(x+), F_{\ell}(x)\right)=F_{\ell}(x)$ and, from this, $N_{0}=\left(M_{0}\right)_{u}=\left(F_{\ell}\right)_{u}=F_{\ell}$, by (4.d).

For the study of the case $t=T$, we first show that given any $x \in \mathbb{R}$, if $\ell(x) \in \operatorname{Cont}(F)$ then $M_{T}(\ell(x))=F(\ell(x))$. If we assume that $[\ell(x), r(\ell(x))] \cap D=\emptyset$ then $M_{T}(x)=\min \left(F(\ell(x)+), F_{\ell}(\ell(x))+T+m / 2\right)=F(\ell(x)+)=F(\ell(x))$. If, on the contrary, $[\ell(x), r(\ell(x))] \cap D \neq \emptyset$ there would exist $d \in D$ such that $\ell(x)<$ $\ell(d) \leqslant d$; but, since $\ell(d)<d$ would imply $\ell(d) \in \operatorname{Cont}(F)$ (due to $\ell(d) \notin D$ and $\ell(\ell(d))=\ell(d))$ it would contradict $d \in D$. Hence $\ell(x)<\ell(d)=d$. From this, $M_{T}(\ell(x))=\min \left(F(\ell(x)+), F_{\ell}(\ell(x))+T+m / 2, F(d-)\right)=F(\ell(x)+)=F(\ell(x))$, because of $F(d-)=F(\ell(d)-)=F_{u}(\ell(d)) \geqslant F(\ell(x)+)$.

Now, we show that $N_{T}(x)=\sup \left\{M_{T}(y): y \leqslant x\right\}$ and $F_{u}(x)=\sup \{F(y): y \leqslant x\}$ are the same number. Clearly, $M_{T}(y) \leqslant F(y+) \leqslant F(y-) \leqslant F_{u}(y) \leqslant F_{u}(x)$ for every $y \leqslant x$, hence $N_{T}(x) \leqslant F_{u}(x)$. Also, given any $\varepsilon>0, F(\ell(x)-)=F_{u}(\ell(x))=$ $F_{u}(x)>F_{u}(x)-\varepsilon$. If $\ell(x) \in \operatorname{Cont}(F)$ then $M_{T}(\ell(x))=F(\ell(x)-)=F(\ell(x)+)>$ $F_{u}(x)-\varepsilon$. If $\ell(x) \notin \operatorname{Cont}(F)$, since $\ell(\ell(x))=\ell(x)$ then $\ell(x) \in D$ and we can take $\delta>0$ such that for $\ell(x)-\delta<y<\ell(x)$ the following is satisfied, $F(y+)>$ $F(\ell(x)-)-\varepsilon, F_{\ell}(y)=F_{\ell}(\ell(x))$ and $\min ([y, r(y)] \cap D)=\ell(x)$. Hence $M_{T}(y)=$ $\min \left(F(y+), F_{\ell}(\ell(x))+T+m / 2, F(\ell(x)-)\right)>F(\ell(x)-)-\varepsilon=F_{u}(x)-\varepsilon$. In both cases then, given any $\varepsilon>0$, there exists $y \leqslant x$ such that $M_{T}(y) \geqslant F_{u}(x)-\varepsilon$ and hence, for any $\varepsilon>0, N_{T}(x) \geqslant F_{u}(x)-\varepsilon$ and, from this, $N_{T}(x) \geqslant F_{u}(x)$.

Lemma 8. For any given $0<t<T$, Disc $(F) \subseteq$ Const $\left(N_{t}\right)$.

Proof: Let $d$ be a point of discontinuity of $F$. Suppose first that $d \in D$; then, since $d \in[d, r(d)]$, we have $M_{t}(d)=\min \left(F(d+), F_{\ell}(d)+t+f(t), F(d-)-m / 2+\right.$ $f(t))=\min \left(F(d+), F_{\ell}(d)+t+f(t)\right)$. Now, since $F(d-)>F(d+)$, there exists $\varepsilon>0$ such that $(d-\varepsilon, d) \subset$ Const $\left(F_{\ell}\right)$ and $(d-\varepsilon, d) \cap D=\emptyset$; hence, $\min ([x, r(x)] \cap D)=d$ for every $x \in(d-\varepsilon, d)$. From this we obtain that $M_{t}(x)=\min \left(F(x+), F_{l}(x)+\right.$ $t+f(t), F(d-)-m / 2+f(t))=\min \left(F(x+), F_{\ell}(d)+t+f(t), F(d-)-m / 2+f(t)\right)$, for every $x \in(d-\varepsilon, d)$. Since $D \neq \emptyset, 0<t<T$ implies that $F(d-)-m / 2+f(t)<$ $F(d-)$; thus, we can assume $\varepsilon$ small enough so as to have $F(x+)>F(d-)-m / 2+$ $f(t)$ for every $x \in(d-\varepsilon, d)$. From all this, $M_{t}(x)=\min \left(F_{\ell}(d)+t+f(t), F(d-)-\right.$ $m / 2+f(t))$ for every $x \in(d-\varepsilon, d)$. In particular, $(d-\varepsilon, d) \subset$ Const $\left(M_{t}\right)$.

Take now $x>d$. Then $L_{t}(x) \leqslant F_{\ell}(x)+t+f(t)$ and $M_{t}(x) \leqslant \min \left(F(x+), F_{l}(x)+t\right.$ $+f(t))$, hence $M_{t}(d+) \leqslant \min \left(F(d+), F_{l}(d)+t+f(t)\right)=M_{t}(d)$. Now, if $F(d+)<$ 
$F_{\ell}(d)+t+f(t)$, then $M_{t}(d-)>M_{t}(d) \geqslant M_{t}(d+)$ which, together with $(d-\varepsilon, d) \subset$ Const $\left(M_{t}\right)$, leads to $d \in$ Const $\left(N_{t}\right)$. If, on the contrary, $F_{\ell}(d)+t+f(t) \leqslant F(d+)$, then $F_{\ell}(d)<F(d+)$ which, in our conditions, implies $d \in \operatorname{Const}\left(F_{\ell}\right)$. Then there exist $\delta>0$ such that $M_{t}(x) \leqslant F_{\ell}(x)+t+f(t)=F_{\ell}(d)+t+f(t)=M_{t}(d)$ for any $d<x<d+\delta$. Therefore, $M_{t}(d-)=M_{t}(d) \geqslant M_{t}(x)$ for all $x \in(d, d+\delta)$, which, together with $(d-\varepsilon, d) \subset$ Const $\left(M_{t}\right)$ leads to $d \in$ Const $\left(N_{t}\right)$.

Finally, suppose $d \notin D$; then $F$ is continuous at $\ell(d)$ (which in particular implies $\ell(d)<d$ ) and $F_{\ell}(\ell(d))=F_{\ell}(d)$. We can then find $\varepsilon>0$ such that $\ell(d)<d-\varepsilon$, $[x, r(x)] \cap D=[d, r(d)] \cap D$ for all $x \in(d-\varepsilon, d+\varepsilon)$ and $F(x+)<F(d-)$ for all $x \in(d, d+\varepsilon)$.

In the case $[d, r(d)] \cap D=\emptyset$, we have $M_{t}(x)=\min \left(F(x+), F_{\ell}(x)+t+f(t)\right)$ for all $x \in(d-\varepsilon, d+\varepsilon)$. Since $M_{t}(\ell(d))=\min \left(F(\ell(d)+), F_{\ell}(\ell(d))+t+f(t)\right)=$ $\min \left(F_{u}(d), F_{\ell}(d)+t+f(t)\right)$ and $F_{u}(d) \geqslant F(x+)$ for all $x \leqslant d$, we obtain $M_{t}(\ell(d)) \geqslant$ $M_{t}(x)$ for all $x \in(d-\varepsilon, d]$. If $F(d+)<F_{\ell}(d)+t+f(t)$ then we can take $\varepsilon$ small enough so as to have $F(x+)<F_{\ell}(d)+t+f(t)$ for all $x \in(d, d+\varepsilon)$, which yields $M_{t}(\ell(d))>$ $M_{t}(x)=F(x+)$ for any $d<x<d+\varepsilon$. If, on the contrary, $F_{\ell}(d)+t+f(t) \leqslant F(d+)$, then $F_{\ell}(d)<F(d+)$ and $d \in \operatorname{Const}\left(F_{\ell}\right)$; hence $M_{t}(x)=\min \left(F(x+), F_{\ell}(d)+t+f(t)\right)$ for all $x \in(d, d+\varepsilon)$, for some $\varepsilon$ small enough. Again, $M_{t}(\ell(d)) \geqslant M_{t}(x)$ for all $x \in(d, d+\varepsilon)$. Thus, in both cases there exists $\varepsilon>0$ such that $M_{t}(\ell(d)) \geqslant M_{t}(x)$ for every $|x-d|<\varepsilon$. Lemma 5 now gives $d \in \operatorname{Const}\left(N_{t}\right)$.

The case $[d, r(d)] \cap \neq \emptyset$ is simpler, since then $d \in$ Const $\left(F_{\ell}\right)$ and there exists $\varepsilon>0$ such that $M_{t}(x)=\min \left(F(x+), F_{\ell}(d)+t+f(t), F\left(d^{\prime}\right)-m / 2+f(t)\right)$ for every $|x-d|<\varepsilon$, where $d^{\prime}=\min ([d, r(d)] \cap D)$. Then as above, $M_{t}(\ell(d)) \geqslant M_{t}(x)$ for every $|x-d|<\varepsilon$ and $d \in \operatorname{Const}\left(N_{t}\right)$.

LEMMA 9. $\left\{N_{t}\right\}_{t \in[0, T]}$ is a family of continuous maps from $\mathbb{R}$ into itself. For any $t \in[0, T]$, the following properties hold.

(9.a) $\operatorname{Cont}(F) \subseteq \operatorname{Cont}\left(M_{t}\right)$.

(9.b) If $x \in \operatorname{Cont}(F)$ and $L_{t}(t)<F(x)$, then $x \in \operatorname{Cont}\left(L_{t}\right)$.

ProOF: In order to prove the continuity of the family of maps $\left\{N_{t}\right\}_{t}$ we only need to show that (9.a) holds; Lemma 8 and the continuity of $N_{0}=F_{\ell}$ and $N_{T}=F_{u}$ then give the desired result. Let $x$ be a point of continuity of $F$. We assume first that $[x, r(x)] \cap D=\emptyset$. We can then find an $\varepsilon>0$ such that $(x-\varepsilon, x+\varepsilon) \cap D=\emptyset$ and $[y, r(y)] \cap D=\emptyset$ for every $y \in(x-\varepsilon, x+\varepsilon)$. Hence $L_{t}(y)=F_{\ell}(y)+t+f(t)$ for every $y \in(x-\varepsilon, x+\varepsilon)$ and, from this, $L_{t}$ and $M_{t}$ are continuous at $x$.

Second, we suppose that $d=\min ([x, r(x)] \cap D)$ and that $x \in \operatorname{Const}\left(F_{\ell}\right)$. We take now $\varepsilon>0$ such that $(x-\varepsilon, x+\varepsilon) \subset$ Const $\left(F_{\ell}\right)$ and $(x-\varepsilon, x+\varepsilon) \cap D=\emptyset$. We then have that $d=\min ([y, r(y)] \cap D)$ for every $y \in(x-\varepsilon, x+\varepsilon)$ and hence 
$L_{t}(y)=\min \left(F_{\ell}(y)+t, F(d-)-m / 2\right)+f(t)=\min \left(F_{\ell}(x)+t, F(d-)-m / 2\right)+f(t)$ for every $y \in(x-\varepsilon, x+\varepsilon)$. Therefore $(x-\varepsilon, x+\varepsilon) \subset$ Const $\left(L_{t}\right)$ and $L_{t}$ and $M_{t}$ are continuous at $x$.

Finally, let $d=\min ([x, r(x)] \cap D)$ and suppose $x \notin \operatorname{Const}\left(F_{\ell}\right)$. Then, by (4.g), $F(x)=F_{l}(x)=F_{\ell}(d) \leqslant F(d+)<F(d-)-m / 2+f(t)$ (due to $D \neq$ $\emptyset)$. We can then find an $\varepsilon>0$ such that $x-\varepsilon<y<x$ implies $[y, r(y)] \cap$ $D=\emptyset$ and $x \leqslant y<x+\varepsilon$ implies $d=\min ([y, r(y)] \cap D)$. Hence, if $x-$ $\varepsilon<y<x$, we obtain $M_{t}(y)=\min \left(F(y+), F_{\ell}(y)+t+f(t)\right)$ and, from this, $M_{t}(x-)=\min \left(F(x), F_{\ell}(x)+t+f(t)\right)=F(x)$. If $x \leqslant y<x+\varepsilon$, we obtain $M_{t}(y)=\min \left(F(y+), F_{\ell}(x)+t+f(t), F(d-)-m / 2+f(t)\right)$; hence $M_{t}(x)=$ $M_{t}(x+)=\min \left(F(x), F_{\ell}(x)+t+f(t), F(d-)-m / 2+f(t)\right)=F(x)$. From all this, $x \in \operatorname{Cont}\left(M_{t}\right)$.

Notice also that in this last case $L_{t}(x)=\min \left(F_{\ell}(x)+t, F(d-)-m / 2\right)+f(t) \geqslant$ $F_{\ell}(x)=F(x)$. Hence, we also obtain (9.b).

Lemma 10. Let $t \in[0, T]$ and $x \in \operatorname{Cont}(F)$. If $N_{t}(x) \neq F(x)$ then $x \in$ Const $\left(N_{t}\right)$.

Proof: From the previous lemma it follows that $x \in \operatorname{Cont}\left(M_{t}\right)$ and $M_{t}(x)=$ $\min \left(F(x), L_{t}(x)\right)$. In the case $F(x) \leqslant L_{t}(x)$ the proof is straightforward, since then $N_{t}(x) \neq F(x)$ implies $N_{t}(x) \neq M_{t}(x)$ and, by (4.g), $x \in$ Const $\left(N_{t}\right)$. We consider now $L_{t}(x)<F(x)$. Because of (9.b) we have that $x \in \operatorname{Cont}\left(L_{t}\right)$ and we can restrict ourselves to a neighbourhood of $x$ such that $L_{t}(y)<F(y+)$ and therefore $M_{t}(y)=L_{t}(y)$.

Thus, if $[x, r(x)] \cap D=\emptyset$ we obtain $F_{\ell}(x) \leqslant F_{\ell}(x)+t+f(t)=L_{t}(x)<F(x)$ and, by (4.g), $x \in \operatorname{Const}\left(F_{\ell}\right)$. Since $x \notin D$, there exists $\varepsilon>0$ such that $(x-\varepsilon, x+\varepsilon) \subset$ Const $\left(F_{\ell}\right)$ and $[y, r(y)] \cap D=\emptyset$ for every $y \in(x-\varepsilon, x+\varepsilon)$. Hence, $M_{t}(y)=L_{t}(y)=F_{\ell}(y)+t+f(t)$ for every $y \in(x-\varepsilon, x+\varepsilon)$, which leads to $x \in$ Const $\left(M_{t}\right) \subseteq$ Const $\left(N_{t}\right)$.

If this is not the case, we set $d=\min ([x, r(x)] \cap D)$. If $F_{l}(x)+t \leqslant F(d-)-m / 2$ then $F_{\ell}(x) \leqslant F_{\ell}(x)+t+f(t)=L_{t}(x)<F(x)$ and we obtain that $x \in \operatorname{Const}\left(F_{\ell}\right)$. If $F(d-)-m / 2<F_{\ell}(x)+t$ then $F(x)>L_{t}(x)=F(d-)-m / 2$ and we can find $a \quad \delta>0$ such that $F(y)>F(d-)-m / 2 \geqslant F(d+)$ for every $|x-y|<\delta$, which, by Lemma 5 , implies $x \in$ Const $\left(F_{\ell}\right)$. Thus, in both cases we can take a small enough $\varepsilon>0$ such that for every $y \in(x-\varepsilon, x+\varepsilon)$ we have $d=\min ([y, r(y)] \cap D)$ and therefore $M_{t}(y)=L_{t}(y)=\min \left(F_{\ell}(x)+t, F(d-)-m / 2\right)+f(t)$. From this, $x \in$ Const $\left(M_{t}\right) \subseteq$ Const $\left(N_{t}\right)$.

We are now prepared to prove our main result.

Proof of Theorem 1: We construct the map $\mathcal{N}:[0, T] \rightarrow \mathbb{R}, t \mapsto \rho\left(N_{t}\right)$. Thanks to Lemma 7 and Lemma 2, this map is continuous since $N_{t}$ is, by Lemma 
9, a continuous map for every value of $t$. Since $N_{0}=F_{\ell}$ and $N_{T}=F_{u}$, for any given $r \in\left(\rho\left(F_{\ell}\right), \rho\left(F_{u}\right)\right)$ there exists $t \in[0, T]$ such that $\rho\left(N_{t}\right)=r$. Then, by Lemma 3, $N_{t}$ has an orbit disjoint from Const $\left(N_{t}\right)$ and, if $r$ is a rational number, $N_{t}$ has a cycle mod 1 disjoint from Const $\left(N_{t}\right)$. Since $\operatorname{Disc}(F) \subset$ Const $\left(N_{t}\right)$, Lemma 10 then implies that for any point of this orbit, $F$ and $N_{t}$ take the same value. Therefore this orbit (or cycle $\bmod 1$ ) is a twist orbit for $F$, thanks to the monotonicity of $N_{t}$. And, of course, its rotation number is $\rho\left(N_{t}\right)=r$.

\section{REFERENCES}

[1] L. Alseda, J. Llibre and M. Misiurewicz, 'Combinatorial dynamics and entropy in dimension one'. Preprint 1990 .

[2] R. Bamon, I.P. Malta, M.J. Pacifico and F. Takens, 'Rotation intervals of endomorphisms of the circle', Ergodic Theory Dynamical Systems 4 (1984), 493-498.

[3] A. Chenciner, J.M. Gambaudo and C. Tresser, 'Une remarque sur la structure des endomorphismes de degré 1 du cercle', C.R. Acad. Sci. Paris Sér. I Math. 289 (1984), 145-148.

[4] F. Esquembre, Rotation sets for circle maps and invariant curves in planar transformations, Ph.D. Thesis (University of Murcia, 1991).

[5] F. Esquembre, 'Rotation sets for some non-continuous maps of degree one', Acta Math. Univ. Comenian. 61 (1992), 167-183.

[6] F. Hofbauer, 'Periodic points for piecewise monotonic transformations', Ergodic. Theory Dynamical Systems 5 (1985), 237-256.

[7] R. Ito, 'Rotation sets are closed', Math. Proc. Cambridge Philos. Soc. 89 (1981), 107-111.

[8] M. Misiurewicz, 'Rotation intervals for a class of maps of the real line into itself', Ergodic. Theory Dynamical Systems 6 (1986), 117-132.

[9] S. Newhouse, J. Palis and F. Takens, 'Bifurcations and stability for families of diffeomorphisms', in Publications Mathématiques 57 (Institute des Hautes Etudes Scientifiques, Paris, 1983), pp. 5-71.

[10] F. Rhodes and C.L. Thompson, 'Rotation numbers for monotone functions of the circle', J. London Math. Soc. 34 (1986), 360-368.

[11] W. Rudin, Principles of Mathematical Analysis (McGraw-Hill, Singapore, 1976).

Department of Mathematics

University of Murcia

30071 Murcia

Spain 